\title{
Gastroscopy-Induced Pancreatitis: A Rare Cause of Post- Procedure Abdominal Pain
}

\author{
Neesa Fadaee ${ }^{a, b}$, Stefaan De Clercq ${ }^{a}$
}

\begin{abstract}
A 52-year-old man presented to the emergency department a few hours post gastroscopy with biopsies of the second part of the duodenum (D2) and colonoscopy with severe acute abdominal pain. On examination he had peri-umbilical tenderness. Subsequent laboratory and biochemical investigations revealed acute pancreatitis. The patient went on to develop complications of acute pancreatitis including bilateral pleural effusions and ascites. He was managed conservatively and was discharged home on day 16 of admission with a plan for elective laparoscopic cholecystectomy in the future.
\end{abstract}

Keywords: Pancreatitis; Complications post gastroscopy; Endoscopic procedure

\section{Introduction}

Pancreatitis is a rare side effect following gastroscopy without ampullary cannulation. It is not well documented in literature with only five case reports published previously. There have been multiple hypotheses relating to etiology. In this case an ampullary cannulation-like effect from a biopsy of a polyp in the second part of the duodenum (D2) resulted in severe acute pancreatitis.

\section{Case Report}

A 52-year-old man underwent a routine diagnostic gastroscopy and colonoscopy following two positive fecal occult blood tests in addition to intermittent symptoms of gastro-esophageal reflux. He was otherwise healthy and had no past medical history of note. He rarely consumed alcohol and was a non-smoker. He did not take any regular medications.

Sedation was initiated with a typical combination of mida-

Manuscript submitted April 21, 2019, accepted April 29, 2019

${ }^{\mathrm{a}}$ General Surgical Department, Gladstone Hospital, Gladstone, QLD, Australia ${ }^{b}$ Corresponding Author: Neesa Fadaee, General Surgical Department, Gladstone Hospital, Gladstone, QLD 4680, Australia.

Email: neesafadaee@gmail.com

doi: https://doi.org/10.14740/jmc3304 zolam and fentanyl, and further sustained with propofol. A total of $60 \mathrm{mg}$ of propofol was required during the procedure. The gastroscopy was completed first with the scope passing easily to D2. He was noted to have a 3-cm sliding hiatus hernia, inflamed gastro-esophageal junction and his Z-line was at $36 \mathrm{~cm}$. No specific abnormality was noted in the stomach. A small polypoid nodule was noted in D2, just proximal to the ampulla of Vater, which was biopsied. Histopathological examination of this lesion was unremarkable and was reported as normal duodenal mucosa.

He was then repositioned and a colonoscopy was performed. The endoscope was easily advanced through to the cecum. He was noted to have a few scattered sigmoid diverticuli and four polyps were resected from his sigmoid and rectum, including a 10-mm pedunculated polyp in the proximal sigmoid colon, $2 \times 5 \mathrm{~mm}$ sessile polyps in the distal sigmoid colon, and a $10-\mathrm{mm}$ pedunculated polyp in the rectum, where the area was marked with a spot marker. Histopathological examination of all four polyps demonstrated an appearance consistent with tubular adenoma.

The patient's post-operative course was unremarkable, with no significant variation in his vital signs noted. Prior to discharge, the patient was tolerating an oral diet. Discharge occurred approximately $2 \mathrm{~h}$ post-procedure. The patient developed severe abdominal pain $3 \mathrm{~h}$ later and subsequently represented to hospital via ambulance services. Additionally, the patient was profoundly hypotensive with a systolic blood pressure of $70 \mathrm{~mm} \mathrm{Hg}$. Fluid resuscitation was administered to obtain good effect with the systolic blood pressure improving to $115 \mathrm{~mm} \mathrm{Hg}$ following $500 \mathrm{~mL}$ fluid bolus. Intravenous opioid analgesia was also provided. The patient reported additional symptoms of diaphoresis and dizziness.

On physical examination the patient's vital signs were as follows: respiratory rate 20 breaths per minute, $\mathrm{SaO} 298 \%$ room air, heart rate 67 beats per minute and blood pressure 115/70 mm Hg. He was afebrile. His cardiovascular and respiratory examinations were unremarkable. Abdominal examination did not demonstrate any significant distension, peritonitis or organomegaly. Umbilical tenderness was noted, in addition to voluntary guarding. There was no percussion or rebound tenderness, and Murphy's sign was negative.

Laboratory investigations revealed a neutrophilia of $11.25 \times$ $10^{9} / \mathrm{L}$ in addition to a raised lipase of 7,050 U/L (normal range $<60 \mathrm{U} / \mathrm{L}$ ). All other hematological and biochemical parameters were within normal limits. The patient underwent a computed tomography (CT) of his abdomen and pelvis as part of his workup in emergency department (ED) to exclude perforation of viscus. It revealed mildly edematous pancreatic head with moderate per- 


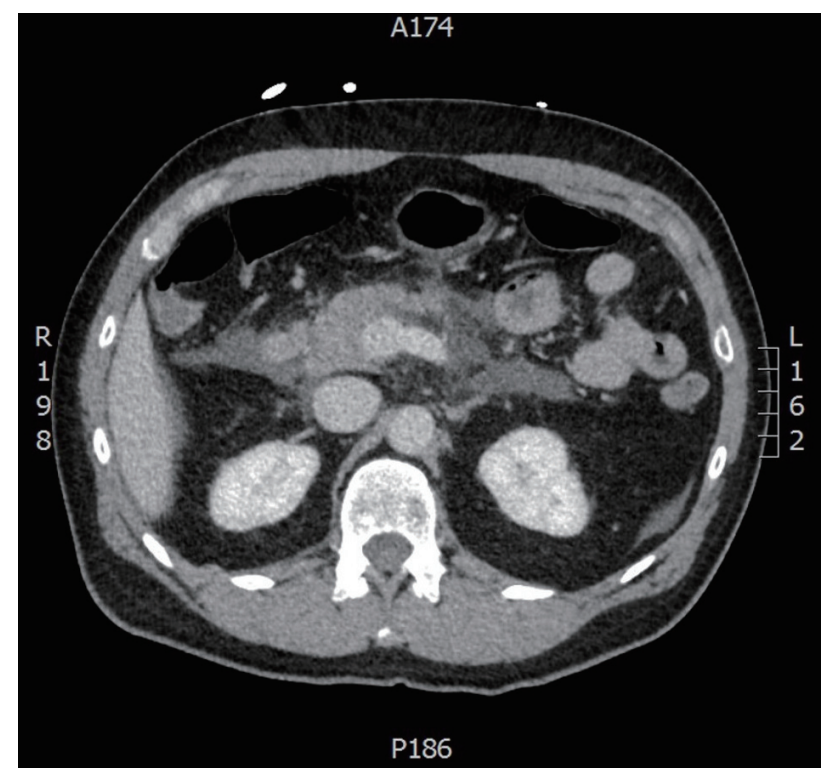

Figure 1. Pancreatic head is mildly edematous with moderate peripancreatic fat stranding and fluid tracking along ridges fascia bilaterally. Appearances are compatible with post endoscopy pancreatitis.

ipancreatic fat stranding and fluid tracking along the ridges fascia bilaterally, compatible with acute pancreatitis as seen in Figure 1.

The patient was managed conservatively with bowel rest, intravenous fluids and analgesia. He required admission to the high dependency unit and the institution of both a ketamine infusion and morphine patient-controlled analgesia device. An abdominal ultrasound was performed which demonstrated an appearance consistent with acute pancreatitis with evidence of pancreatic steatosis and acute inflammation. He was found to have biliary sludge. There was no evidence of cholecystitis and his common bile duct was not dilated measuring $4 \mathrm{~mm}$ in diameter.

Despite conservative measures the patient developed acute complications of pancreatitis including a pancreatic ileus and bilateral pleural effusions. Due to lack of clinical improvement a repeat $\mathrm{CT}$ abdomen and pelvis was conducted on day 5 of admission. It was concerning for a necrotic segment in the body of the pancreas with an increase in the volume of peripancreatic fat stranding and free fluid in relation to the pancreas. An ascitic collection was also noted in the pelvis as seen in Figure 2.

Advice was sought from the hepatobiliary team at a tertiary referral centre who suggested ongoing conservative management with interval imaging. A repeat CT scan was conducted on day 13 of admission which revealed an evolving pancreatitis with maturation of peripancreatic fluid which was not loculated. There was a mid-body pancreatic pseudocyst developing with resolution of pelvic fluid and pleural effusions. With ongoing clinical improvement, the patient was discharged home on day 16 with a plan for potential elective cholecystectomy.

\section{Discussion}

Gastroscopy and colonoscopy are commonly performed diagnostic and therapeutic procedures $[1,2]$. Complications include

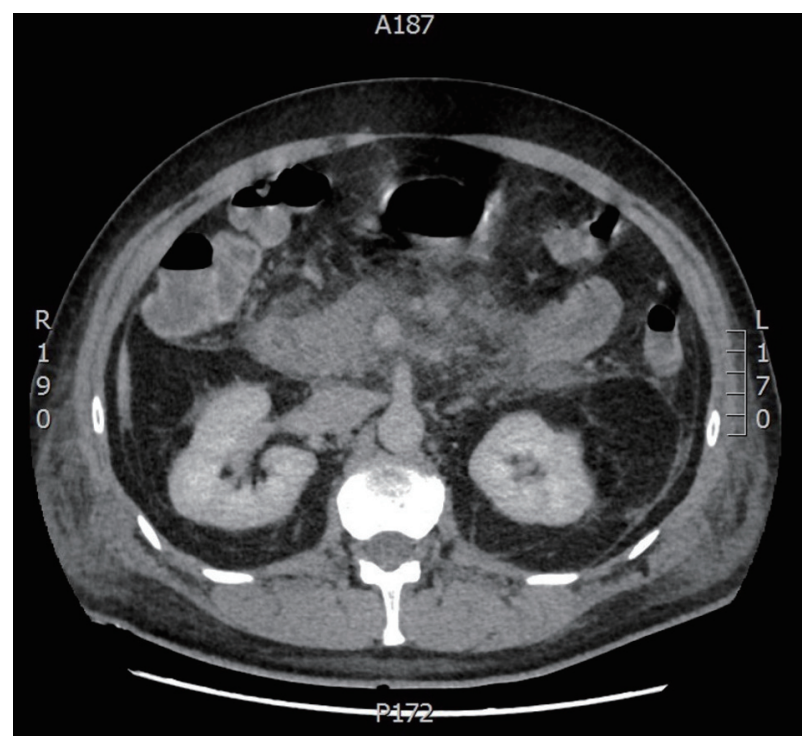

Figure 2. Segment of low attenuation of the body of the pancreas concerning for necrotic area. Increase in diffuse peripancreatic stranding with development of free fluid collections in relation to the pancreas.

hemorrhage post biopsy and polypectomy, perforation and the associated effects of procedural sedation [1, 3, 4]. Although pancreatitis is a well-documented complication of some endoscopic procedures, such as endoscopic retrograde cholangiopancreatography (ERCP), it is not commonly observed following gastroscopy [5]. This is presumably due to the fact that ampullary cannulation is the etiological trigger for inflammation, and, naturally, this does not occur during a gastroscopy [6].

In this case the temporal relationship between the endoscopic procedure and the onset of pancreatitis suggests an etiological relation rather than mere coincidence [6]. Additionally, the patient had minimal risk factors for pancreatitis, besides biliary sludge. However, as noted previously, there was no common bile duct (CBD) dilatation evident on imaging. There was no evidence to suggest excessive alcohol consumption, hypercalcemia or hypercholesterolemia.

It is postulated that in this case, the development of pancreatitis has occurred secondary to the biopsy of the penduculated polyp observed in D2. Given the proximity of this polyp to the ampulla of Vater, it is thought that the inflammation associated with the trauma of the biopsy has spread into the ampulla and caused an effect akin to ampullary cannulation.

Pancreatitis post gastroscopy is not a well-documented side effect [6-8]. In addition to this case there are only five documented cases of pancreatitis post gastroscopy without ampullary cannulation [3,5-8] and only three cases post colonoscopy $[7,9,10]$.

It has been hypothesised that a variety of factors may lead to local inflammation and pancreatitis, including local trauma from the duodenum pushing on the head of the pancreas, excessive duodenal insufflation, and direct trauma from the colonoscopy traversing the transverse colon and splenic flexure $[5,7]$. Whatever the cause, it appears that pancreatitis is a rare complication of gastrointestinal endoscopy and should be considered as a differential diagnosis for post-endoscopy 
abdominal pain once more common causes are excluded.

\section{Acknowledgments}

None to declare.

\section{Financial Disclosure}

None to declare.

\section{Conflict of Interest}

None to declare.

\section{Informed Consent}

Informed consent was obtained from patient prior to completing case report.

\section{Author Contributions}

Both authors contributed to the conception, writing and editing of the case report. NF was involved in the acquisition, analysis and interpretation of data. Both authors agree to be accountable for all aspects of the work.

\section{References}

1. Habr-Gama A, Waye JD. Complications and hazards of gastrointestinal endoscopy. World J Surg. 1989;13(2):193-201.

2. Berger JC, Muzaale AD, James N, Hoque M, Wang JM, Montgomery RA, Massie AB, et al. Living kidney donors ages 70 and older: recipient and donor outcomes. Clin J Am Soc Nephrol. 2011;6(12):2887-2893.

3. Deschamps JP, Allemand H, Janin Magnificat R, Camelot G, Gillet M, Carayon P. Acute pancreatitis following gastrointestinal endoscopy without ampullary cannulation. Endoscopy. 1982;14(3):105-106.

4. Levy I, Gralnek IM. Complications of diagnostic colonoscopy, upper endoscopy, and enteroscopy. Best Pract Res Clin Gastroenterol. 2016;30(5):705-718.

5. Nevins AB, Keeffe EB. Acute pancreatitis after gastrointestinal endoscopy. J Clin Gastroenterol. 2002;34(1):9495.

6. Wu P, Hubbard H. Acute pancreatitis after upper endoscopy. Case Rep Intern Med. 2016;3(4):41-43.

7. Ko HH, Jamieson T, Bressler B. Acute pancreatitis and ileus post colonoscopy. Can J Gastroenterol. 2009;23(8):551-553.

8. Nwafo NA. Acute pancreatitis following oesophagogastroduodenoscopy. BMJ Case Rep. 2017;2017.

9. Shekhar S, Singh S, Gupta S. Postcolonoscopy pancreatitis. Endoscopy. 2014;46(Suppl 1 UCTN):E515-516.

10. Khashram M, Frizelle FA. Colonoscopy - a rare cause of pancreatitis. N Z Med J. 2011;124(1345):74-76. 